\title{
TRIGGER CONTROL AND FAULT REACTION CIRCUITRY FOR THE SOLID-STATE SWITCH MODULATOR DECK AT THE MIT-BATES S-BAND TRANSMITTER
}

\author{
R. Campbell, A. Hawkins, W. North (Consultant), L. Solheim, C. Wolcott", \\ A. Zolfaghari, MIT Bates
}

\section{Abstract}

This paper describes the trigger control and fault reaction circuitry for the new solid-state switch modulator at the MIT Bates Linear Accelerator Center. This new circuitry has been designed and built to control the new cathodeswitching solid-state modulator that replaces the old vacuum-tube technology modulator. The old modulator used a start signal to commence its pulses and a separate stop signal to end its pulses. The new system uses a single gate signal to control the modulator pulse.

The trigger control circuit is a stand-alone control unit that can operate in a local (manual) mode or a remote mode. In the local mode the unit uses its own oscillator to run the transmitter. In the remote mode the acceleratorcontrol computer can turn triggers on or off (enable the triggers), can reset the unit, and can send the gate signal that triggers the modulator. There is no microprocessor fault control of the transmitter. The trigger control chassis receives all necessary signals from the transmitter and performs all necessary trigger control and fault reaction functions by itself. These fault reactions can be to turn off the solid-state switch, to fire the crowbar, or simply to light LEDs. The main accelerator-control microprocessor receives report signals from the trigger control unit to alert the accelerator operators to the status of the transmitter.

The trigger control circuitry limits the modulator pulse width to 5 microseconds longer than the gate signal up to a maximum of 55 microseconds and limits the pulse repetition frequency to under $2 \mathrm{kHz}$. All the circuitry is designed with noise suppression techniques and with a high level of noise immunity ( 5 volts or more) to ensure faultfree operation in a noisy, transient-filled environment.

\section{INTRODUCTION}

The MIT-Bates Linear Accelerator is powered by six RF transmitter stations that each contain two RF amplifying klystrons. The old modulator design, which pulses highvoltage across the klystrons, uses vacuum-tube technology that is prone to failures and is difficult to maintain. This design consists of a center-deck and two outboard switchtube decks that each hold two parallel-connected L-5097 Beam Switch Tubes (BSTs) that are in series with each klystron and the High-Voltage Power Supply (HVPS) output. The center deck contains the power supplies and various vacuum-tube power-circuitry that sends highvoltage pulses to the BSTs modulating anodes. These pulses "turn on" the BSTs to the desired current-regulation level and allow a current pulse to be sent through each klystron. The klystron current pulses are initiated by a Start trigger signal and cut off by a Stop trigger signal. The new modulator system creates two separate modulators, one for each klystron, using the existing switch-tube decks and BSTs. The center deck is eliminated. At the heart of the new design is a $20 \mathrm{kV}, 100 \mathrm{Amp}$ solid-state switch (built by Diversified Technology, Inc. in Bedford, MA) which is connected to the parallel BSTs in a cathode switching configuration. A $0-20 \mathrm{kV}$ DC power supply is connected between the negative high voltage of the HVPS and the modulating anodes of the BSTs to set them for the desired current pulse amplitude. A fiber-optic gate signal is used to close the switch. When light is transmitted, the switch closes until the light is removed. With the closing of the switch, the cathodes of the BSTs are taken to the negative highvoltage $(-180 \mathrm{kV})$ of the transmitter and the klystrons are pulsed. When the switch opens, the BSTs stop conducting, thereby blocking the current through the klystrons.

Because of the basic differences between the two systems, and due to new fault monitoring devices, a new approach was needed to trigger the new modulator. Therefore, a new trigger control chassis was designed and a prototype built. The control circuitry was designed at Bates and ModPower, Inc. in Taunton, MA designed the power supply and built the complete prototype system. The system contains its own power supply, power monitoring, remote and local trigger control, trigger filtering, modulator fault detection and latches, crowbar control, and fail-safe safety features. The circuitry is designed with noise suppression techniques with a high level of noise immunity ( 5 volts or more) to ensure fault-free operation in a noisy, transient-filled environment.

\section{POWER SUPPLY}

The main power to the chassis is $120 \mathrm{VAC}$. The AC input is first fused then bypassed from line to neutral by back to back zener diodes and a $1 \mu \mathrm{F}$ capacitor (cap). The AC is then filtered with a line filter. The filter output is bypassed from line to neutral by a $1 \mu \mathrm{F}$ cap, back to back zener diodes

\footnotetext{
\# Email: CWOLCOTT@MIT.EDU
} 
and a 100k resistor. The line filter output from line to ground is bypassed by a $4.7 \mathrm{nF}$ cap as is the output from neutral to ground. A power switch switches the $\mathrm{AC}$ on to the power supply. The power supply outputs are $+5 \mathrm{VDC}$, $+7.5 \mathrm{VDC},+10 \mathrm{VDC},+12 \mathrm{VDC},+15 \mathrm{VDC}$, and +20VDC. These voltages are used to power Integrated Chips (ICs), pulse fiber-optic transmitters, and as reference voltages for Comparators (LM339s). Comparators were used because of their high noise immunity features. Each DC voltage is produced from a separate fused transformer whose output is full-wave rectified and then filtered. An adjustable regulator with an adjustable reference set by a 12-turn potentiometer (pot) sets the voltages precisely. Every IC power supply input travels through a ferrite bead and is bypassed by a $0.1 \mu \mathrm{F}$ and $10 \mu \mathrm{F}$ cap. The power supply is self-monitoring and if any DC voltage fails, the crowbar is fired and the modulators are latched off, stopping all transmitter operation. If this occurs a red LED is lit to show which voltage has failed. As with any fault the trigger control needs to be reset (either locally or by remote) to resume operation. As long as the power supply is functioning properly a green LED is lit to indicate a good power supply.

\section{TRIGGER CONTROL}

The primary function of the trigger control chassis is to gate the modulator on and off in a controlled fashion. This can be done by two sources: a remote gate signal or a gate signal from the local oscillator. A front panel break-beforemake switch labeled Remote/Local selects the trigger source. If the remote source is selected (a computer generated signal transmitted from the control room via fiber optic cable) the control room's gate signal will run the modulator. The remote trigger is input to the circuitry through a pulse transformer to ensure galvanic isolation. The gate signal then undergoes two filtering processes. The first filter blocks any pulse that is longer than $55 \mu$ s. If any remote gate signal longer than $55 \mu$ s is sent it is cut off at $55 \mu \mathrm{s}$, the modulator is latched off, and an LED is lit to show that a remote width fault has occurred. The second filter is a pulse repetition frequency (PRF) filter. It blocks any frequencies higher that $2 \mathrm{kHz}$. If any pulse is detected within $500 \mu$ s of another $(2 \mathrm{kHz})$, the pulse is blocked, the modulator is latched off, and a red LED is lit to indicate a remote PRF fault. If a gate signal gets past these filters it passes through the Remote/Local switch and then to the Enable Triggers circuit. The transmitter system has many interlocks that must be satisfied before the main highvoltage can be turned on. If all these interlocks are satisfied, and high voltage is turned on, a 24 VDC signal is sent to the trigger control chassis. If this signal is not present the triggers cannot be enabled and the gate signal cannot get past the enable circuit. If the 24VDC signal is present, a green LED labeled "Ready for Triggers" is lit. Only then can the triggers be enabled, either remotely or by a local pushbutton. When the triggers have been enabled the gate can pass through the enable circuit to gate a transistor. This transistor switches 15 VDC to a pair of parallel-connected fiber-optic transmitter circuits (one for each modulator). In each path are five fiber optic transmitters (one for each module of the solid-state switch), a resistor, and a switch that allows for the manual shut-off of either modulator so that one klystron can be pulsed while the other is not used. When the gate signal switches the transistor on, current flows through the fiber optic transmitter LEDs. The lights are transmitted via fiber optic cables to the solid state switch. The switch closes and pulses current through the klystron. At the bottom of each fiber-optic transmitter string is a viewing resistor that develops a voltage whenever current is flowing through the fiber-optic transmitters. A front panel BNC bulkhead is connected across each resistor to allow monitoring of the trigger signals. This signal is also connected to a panelmounted meter that displays the PRF of the modulators, and is also sent to the switch-status fault detection circuit discussed below.

If the local oscillator is selected to gate the modulator, the signal is taken from an oscillator circuit inside the chassis. This circuit supplies a gate that is variable from 3-55 $\mu$ s long with a PRF variable from $0-2 \mathrm{kHz}$. These parameters are controlled by panel mounted potentiometers.

\section{FAULT DETECTION}

The trigger control chassis must monitor many modulator signals to protect the modulator and klystrons from system faults. Besides the remote width, remote PRF, and power supply faults already discussed the system monitors each modulator's BST difference current, each klystron's body current and collector current, and the total ground current of the entire transmitter. These signals are sent from current monitor transformers via coaxial cables that pass through pulse baluns to attenuate any shield noise. The circuitry also monitors the status of each module in both solid-state switches to verify its operation.

As stated, each modulator deck contains two parallel-connected BSTs. If one of the BSTs arcs there will be a huge difference between the current in the arcing tube and the current in the non-arcing tube. This difference current is monitored, and if a large enough difference is detected the system crowbar is fired and the triggers are disabled. The collector current of each klystron is compared to a width window that is the original gate signal plus $5 \mu$ s. If any collector current in either klystron is detected outside this window, indicating a wide pulse or spontaneous current, the crowbar is fired and the triggers are latched off. The body current of each klystron is monitored and if either exceeds 10 Amps the triggers are latched off. In this way, if a klystron arcs, but the BSTs do not, the klystron arc can be extinguished by simply shutting off the solid-state switch. The body current signal will be a very useful new tool. The total ground current fault is more 
complicated. Because it is desirable not to fire the crowbar in the event of a klystron arc, the ground current fault detection circuit must be slowed down slightly. A klystron arcs to ground and this shows up as both klystron body current and ground current. Therefore, if the ground current detection circuit is allowed to fire the crowbar instantly, the switch will not have time to extinguish the arc. So a delay of $10 \mu \mathrm{s}$ was introduced into the ground current detection circuit. However, if the detected ground current is still present after the $10 \mu$ s delay, indicating a problem other than a klystron arc, the crowbar is fired. All of these fault detection circuits have their own latches and LEDs that will indicate what fault occurred. Each circuit latches a set of relay contacts which alerts the control room computer to the fault condition. Each one of these signals can be monitored by an oscilloscope from front panel BNC connections. Another set of signals that is monitored is the switch status signals from the solid-state switch. The switch contains 5 switch modules connected in series. Each module sends back a fiber-optic signal indicating if it is open or closed. Every switch status signal is compared to each gate signal to check for proper operation. If a fault occurs, an LED is lit to indicate which module is bad and an overall switch fault signal is sent to the control room by a relay contact.

\section{CROWBAR CONTROL}

The crowbar control chassis receives a signal from the trigger control chassis and then sends a high-voltage pulse $(15 \mathrm{kV})$ to a large step-up pulse transformer which fires the system crowbar and dumps the high-voltage of the transmitter. Because the old crowbar control chassis will be replaced eventually, two crowbar firing circuits were built into the trigger control chassis; one for the old crowbar chassis and one for the new. The circuit for the old crowbar chassis sends a 15VDC pulse. Because the old system requires a pulse it was not possible to make it fail-safe. The new system, however, will be fail safe. A current sinking field-effect transistor (FET) will hold a voltage generated in the new crowbar chassis down through an interconnecting cable. If the cable is removed, if the trigger control chassis is shut off, or if the FET drive fails the crowbar will fire rendering the system safe for equipment and personnel. In the event of a fault requiring a crowbar the FET is turned off and the crowbar will fire. A Crowbar Test button is mounted on the front panel that sends a high signal to all the fault detection circuits and not only fires the crowbar but tests all the latches as well.

\section{FAIL-SAFE FEATURES}

The trigger control circuitry was designed to be as fail safe as possible. When the unit is turned on or off, all the faults are set to ensure that no spurious signals occur. All the current transformers inside the transmitter actually sink voltage because of their low impedance. If either end of any coaxial cable that carries fault signals is disconnected, the trigger control will act as if that fault occurred; either by firing the crowbar or shutting of the triggers. Also, every comparator, transistor, latch, or logic gate in any fault circuit sinks current or voltage in its "good" condition. If any IC is removed, loses power, or if any connection is misaligned or broken, the circuit will act as though that fault had occurred.

\section{CONCLUSION}

Because the old modulator has become obsolete and unreliable and has proven to be too difficult to maintain, we have the chance to greatly improve the entire transmitter. Simplicity, safety, and reliability have driven the new modulator and trigger control system design. An entirely new strategy for running the transmitter will be implemented. The control circuitry is noise free, robust, and fail-safe. It uses old and new modulator signals that will allow for better fault control strategies, better monitoring and easier troubleshooting. It has been designed to interface with a central computer but does not depend on a microprocessor or software for equipment and personnel safety. The combination of a new modulator system with the new trigger control circuitry will replace obsolete and failing technology and significantly improve the reliability and performance of the accelerator and the RF transmitters. 